\title{
The gamma-ray emitting radio-loud narrow-line Seyfert 1 galaxy PKS 2004-447
}

\section{The radio view ${ }^{\star}$}

R. Schulz ${ }^{1,2}$, A. Kreikenbohm ${ }^{1,2}$, M. Kadler ${ }^{1}$, R. Ojha ${ }^{3,4,5}$, E. Ros ${ }^{6,7,8}$, J. Stevens ${ }^{9}$, P. G. Edwards ${ }^{9}$, B. Carpenter ${ }^{3,5}$, D. Elsässer ${ }^{1}$, N. Gehrels ${ }^{3}$, C. Großberger ${ }^{10,2}$, H. Hase $^{11}$, S. Horiuchi ${ }^{12}$, J. E. J. Lovell ${ }^{13}$, K. Mannheim ${ }^{1}$, A. Markowitz ${ }^{2,14, \star \star}$, C. Müller ${ }^{15,2}$, C. Phillips ${ }^{9}$, C. Plötz ${ }^{11}$, J. Quick ${ }^{16}$, J. Trüstedt ${ }^{1}$, A. K. Tzioumis ${ }^{9}$, and J. Wilms ${ }^{2}$

${ }^{1}$ Lehrstuhl für Astronomie, Universität Würzburg, Campus Hubland Nord, Emil-Fischer-Str. 31, 97074 Würzburg, Germany e-mail: robert.schulz@physik.uni-wuerzburg.de

2 Dr. Remeis-Sternwarte \& ECAP, Universität Erlangen-Nürnberg, Sternwartstr. 7, 96049 Bamberg, Germany

3 NASA, Goddard Space Flight Center, 8800 Greenbelt Rd, Greenbelt, MD 20771, USA

4 University of Maryland, Baltimore County, 1000 Hilltop Cir, Baltimore, MD 21250, USA

5 The Catholic University of America, 620 Michigan Ave NE, Washington, DC 20064, USA

${ }^{6}$ Max-Planck-Institut für Radioastronomie, Auf dem Hügel 69, 53121 Bonn, Germany

7 Observatori Astronòmic, Univ. València, 46980 Paterna València, Spain

8 Dept. Astronomia i Astrofissica, Univ. València, 46100 Burjassot, València, Spain

9 CSIRO, Astronomy and Space Science, ATNF, PO Box 76 Epping, NSW 1710, Australia

10 Max-Planck-Institut für extraterrestrische Physik, Giessenbachstrasse 1, 85741 Garching, Germany

11 Bundesamt für Kartographie und Geodäsie, 93444 Bad Kötzingen, Germany

12 CSIRO Astronomy and Space Science, Canberra Deep Space Communications Complex, PO Box 1035, Tuggeranong ACT 2901, Australia

13 School of Mathematics \& Physics, University of Tasmania, Private Bag 37, Hobart, 7001 Tasmania, Australia

${ }^{14}$ University of California, San Diego, CASS, 9500 Gilman Dr., MC 0424, La Jolla, CA 92093-0424, USA

15 Department of Astrophysics/MAPP, Radboud University Nijmegen, PO Box 9010, 6500 GL Nijmegen, The Netherlands

16 Hartebeesthoek Radio Astronomy Observatory, 1740 Krugersdorp, South Africa

Received 18 September 2015 / Accepted 25 October 2015

\section{ABSTRACT}

Context. $\Gamma$-ray-detected radio-loud narrow-line Seyfert 1 ( $\gamma$-NLS1) galaxies constitute a small but interesting sample of the $\gamma$-ray-loud AGN. The radio-loudest $\gamma$-NLS1 known, PKS 2004-447, is located in the southern hemisphere and is monitored in the radio regime by the multiwavelength monitoring programme TANAMI.

Aims. We aim for the first detailed study of the radio morphology and long-term radio spectral evolution of PKS 2004-447, which are essential for understanding the diversity of the radio properties of $\gamma$-NLS1s.

Methods. The TANAMI VLBI monitoring program uses the Australian Long Baseline Array (LBA) and telescopes in Antarctica, Chile, New Zealand, and South Africa to monitor the jets of radio-loud active galaxies in the southern hemisphere. Lower resolution radio flux density measurements at multiple radio frequencies over four years of observations were obtained with the Australia Telescope Compact Array (ATCA).

Results. The TANAMI VLBI image at $8.4 \mathrm{GHz}$ shows an extended one-sided jet with a dominant compact VLBI core. Its brightness temperature is consistent with equipartition, but it is an order of magnitude below other $\gamma$-NLS1s with the sample value varying over two orders of magnitude. We find a compact morphology with a projected large-scale size $<11 \mathrm{kpc}$ and a persistent steep radio spectrum with moderate flux-density variability.

Conclusions. PKS 2004-447 appears to be a unique member of the $\gamma$-NLS1 sample. It exhibits blazar-like features, such as a flat featureless X-ray spectrum and a core-dominated, one-sided parsec-scale jet with indications for relativistic beaming. However, the data also reveal properties atypical for blazars, such as a radio spectrum and large-scale size consistent with compact-steep-spectrum (CSS) objects, which are usually associated with young radio sources. These characteristics are unique among all $\gamma$-NLS1s and extremely rare among $\gamma$-ray-loud AGN.

Key words. galaxies: active - galaxies: individual: PKS 2004-447 - radio continuum: galaxies - techniques: interferometric galaxies: jets

\footnotetext{
* The VLBI images shown in Figs. 3 and 4 (as FITS files) and the ATCA data listed in Table A.1 are available at the CDS via anonymous ftp to cdsarc.u-strasbg. fr $(130.79 .128 .5)$ or via http://cdsarc.u-strasbg.fr/viz-bin/qcat?J/A+A/588/A146

$\star \star$ Alexander von Humboldt Fellow.
}

\section{Introduction}

The radio-loud versions of narrow-line Seyfert 1 (RL-NLS1) galaxies have attracted growing interest in recent years, especially since Abdo et al. (2009a) reported detecting $\gamma$-ray emission from PMN J0948+0022 with the LAT instrument on-board the Fermi $\gamma$-ray satellite (Atwood et al. 2009). NLS1 galaxies 
harbour an active galactic nucleus (AGN) that exhibits both broad and narrow emission lines as in typical type-1 AGNs. The broad lines, however, are substantially narrower than in other type-1 AGNs. NLS1 galaxies show strong and narrow $\mathrm{H} \beta$ emission with a flux ratio of $\mathrm{O}[\mathrm{III}] / \mathrm{H} \beta \leq 3$, a full width at half maximum of broad $\mathrm{H} \beta_{\mathrm{FWHM}} \leq 2000 \mathrm{~km} \mathrm{~s}^{-1}$, and strong Fe II emission (Osterbrock \& Pogge 1985). It is common for NLS1s to show variability in flux and photon index (e.g., Boller et al. 1997). Like quasars, they can be divided into radio-quiet and radio-loud categories, where the radio-loudness $\mathrm{RL}_{v}$ is defined as the ratio of the radio flux density at frequency $v$ to the optical flux at $440 \mathrm{~nm}$. A source is commonly characterized as radio-loud when $\mathrm{RL}_{1.4 \mathrm{GHz}}>10$.

RL-NLS1 galaxies are rare among radio-loud AGN (Komossa et al. 2006; Zhou et al. 2006). However, the total number of known RL-NLS1s has increased in recent years. The first dedicated study and search of RL-NLS1s was performed by Komossa et al. (2006), yielding a sample of 11 sources most of which were previously not known to be RL-NLS1s. Then, Yuan et al. (2008) compiled a genuine sample of 23 RL-NLS1s with $\mathrm{RL}_{1.4 \mathrm{GHz}}>100$, with black hole masses of $10^{6}-10^{8} M_{\odot}$ and Eddingtion ratios $R_{\text {Edd }} \approx 1$. Based on these and other studies, Foschini et al. (2015) and Berton et al. (2015) have a combined sample of 60 sources with $\mathrm{RL}_{5 \mathrm{GHz}}>10$. Only a few RL-NLS1s have been detected by Fermi/LAT ( $\gamma$-NLS1, Abdo et al. 2009b; Foschini et al. 2015; D'Ammando et al. 2015; Yao et al. 2015, see Sect. 4.3) with most of them listed in the Third Fermi/LAT Catalog of AGNs with $b<-10^{\circ}$ and $b>10^{\circ}$ (3LAC, Ackermann et al. 2015). The $\gamma$-NLS1 galaxies belong to the exclusive group of so-called non-blazar AGNs in the 3LAC, which make up only about $\sim 2 \%$ of the 3LAC sources. The majority of sources in the 3LAC are blazars, which appear to be hosted by elliptical galaxies with central black hole masses above $10^{8} M_{\odot}$ (e.g., Marscher 2009 and references therein). They have powerful jets aligned close to the line of sight.

PKS 2004-447 is an RL-NLS1 at a redshift of $z=0.24$ (Drinkwater et al. 1997). It stands out from $\gamma$-NLS1s because of its unusual radio properties. With $\mathrm{RL}_{4.85 \mathrm{GHz}}=1700-6300$, depending on the optical flux (Oshlack et al. 2001, hereafter Osh01), it has the highest radio loudness and shows a steep radio spectrum with a spectral index ${ }^{1}$ of $\alpha_{\mathrm{r}}<-0.5$ (Osh01; Gallo et al. 2006, hereafter G06). Observations with the Australia Telescope Compact Array (ATCA) show an unresolved source, suggesting that it can be classified as a compact steep-spectrum (CSS) radio source (Osh01, G06).

PKS 2004-447 exhibits only weak Fe II emission, with $E W_{\mathrm{Fe} \text { II }} \leq 10 \AA$, a flux ratio $\mathrm{O}[\mathrm{III}] / \mathrm{H} \beta=1.6$, and $\mathrm{H} \beta_{\mathrm{FWHM}}=$ $1447 \mathrm{~km} \mathrm{~s}^{-1}$ (Osh01). As a result, its NLS1 classification has been under discussion (e.g., Zhou et al. 2006; Yuan et al. 2008). Despite its steep spectral index, it was included in the sample of flat spectrum RL-NLS1 studied by Foschini et al. (2015).

This is the second in a series of papers presenting results from broadband observations of PKS 2004-447. The observations reported in this series were conducted as part of the TANAMI ${ }^{2}$ multiwavelength programme (Ojha et al. 2010; Kadler et al. 2015) that monitors $\gamma$-ray loud AGNs south of $-30^{\circ}$ declination. In a separate paper (Kreikenbohm et al. 2016, hereafter Paper I), we discussed X-ray observations with

\footnotetext{
1 The spectral index $\alpha$ is defined as $S_{v} \propto v^{\alpha}$, where $S_{v}$ is the flux density at frequency $v$.

2 Tracking Active Nuclei with Austral Milliarcsecond Interferometry http://pulsar.sternwarte.uni-erlangen.de/tanami/ projects/
}

XMM-Newton and Swift, finding a flat blazar-like power law and moderate variability on time scales down to months with an observed luminosity of $(0.7-2.6) \times 10^{44} \mathrm{erg} / \mathrm{s}$. In this paper, we present the first Very-Long-Baseline Interferometry (VLBI) image for PKS 2004-447 at $8.4 \mathrm{GHz}$ (the highest radio frequency VLBI image of the source in the literature so far), as well as the first long-term simultaneous multi-frequency radio monitoring with ATCA.

In the next section, we discuss the data reduction followed by a presentation of our results in Sect. 3. In Sect. 4, we discuss our findings and compare them within the sample of $\gamma$-NLS1.

We adopt a $\Lambda$ CDM cosmology, with $H_{0}=70 \mathrm{~km} \mathrm{~s}^{-1} \mathrm{Mpc}$, $\Omega_{\mathrm{M}}=0.3$, and $\Lambda=0.7$ (Freedman et al. 2001).

\section{Observations and data reduction}

\subsection{ATCA observations}

As part of the TANAMI programme, ATCA has been monitoring $\gamma$-ray-detected AGN at frequencies between $\sim 5 \mathrm{GHz}$ and $40 \mathrm{GHz}$ since 2007 (Stevens et al. 2012). PKS 2004-447 has been included in the programme since 2011 May 17. Each observing frequency is the centre of a $2 \mathrm{GHz}$ wide band. PKS 1934-638 has been used as a flux density calibrator. Table A.1 lists the data obtained until 2014 Jun. 13 with statistical errors. In addition, we make use of monitoring data from the ATCA Calibrator Database ${ }^{3}$, extending the spectral coverage at selected epochs to $1.7 \mathrm{GHz}$ and $45 \mathrm{GHz}$.

ATCA observations of this source show a moderate defect ${ }^{4}$ and a very low closure phase at $17 \mathrm{GHz}$ and above. The closure phase is an interferometric quantity that is independent of errors introduced at any individual array element, and it will always be zero (to within the noise of the measurement) if an unresolved source is the dominant source of the flux density in the field of view. The closure phase will deviate from zero if there are other sources producing significant flux density within the field.

The defect uses the difference between the scalar and vectoraveraged flux densities to give us more information about the field. Vector averaging uses the phase information obtained by the interferometer to attenuate sources of flux density away from the phase centre of the measurement. The effect of this averaging is to only make a measurement of the flux density of the source that the array is pointing at directly, and this effect is strongest when using widely spaced array elements. Scalar averaging, however, tends to capture a lot more of the flux density being seen by each array element, regardless of where the flux density is being produced within the field of view.

For a field of view that is empty apart from a point source at the phase centre, both the closure phase and defect would be zero to within the noise level of the measurement. A significant defect and a closure phase close to zero would suggest that some amount of flux density is coming from sources that are not at the phase centre, what we call "confusing structure". This structure could affect the calibration, and because this effect depends on frequency, it will tend to make the radio spectrum slightly steeper. However, we consider this to be negligible for our ATCA data. The defect at a frequency of $17 \mathrm{GHz}$ and above indicates that the source is resolved by ATCA. Conversely, the lack of a defect at $9.0 \mathrm{GHz}$ shows that the source is unresolved for the ATCA at this frequency. In combination, the maximum scales of extended emission seem to range between 1."7 and 0.' 8 .

\footnotetext{
3 http://www .narrabri .atnf.csiro.au/calibrators/

4 Defect is defined as $\left(\left[S_{\mathrm{sca}} / S_{\mathrm{vec}}\right]-1\right) \times 100 \%$, where $S_{\mathrm{sca}}$ is the scalaraveraged flux density, and $S_{\text {vec }}$ is the vector-averaged flux density.
} 
R. Schulz et al.: The gamma-ray emitting radio-loud narrow-line Seyfert 1 galaxy PKS 2004-447. II.

Table 1. Details of interferometric observations and image parameters.

\begin{tabular}{|c|c|c|c|c|c|c|c|c|c|}
\hline $\begin{array}{l}\text { Date } \\
\text { [yyyy-mm-dd] }\end{array}$ & $\begin{array}{l}\text { Freq. } \\
\text { [GHz] }\end{array}$ & Array $^{a}$ & $\begin{array}{l}\text { Taper } \\
{[\mathrm{M} \lambda]}\end{array}$ & $\begin{array}{c}S_{\text {peak }} \\
{\left[\mathrm{mJy}_{\text {beam }}{ }^{-1}\right]}\end{array}$ & $\begin{array}{c}\sigma_{\mathrm{rms}} \\
{\left[\mathrm{mJy} \mathrm{beam}^{-1}\right]}\end{array}$ & $\begin{array}{l}S_{\text {total }} \\
{[\mathrm{mJy}]}\end{array}$ & $\begin{array}{l}b_{\text {maj }}^{b} \\
{[\text { mas] }}\end{array}$ & $\begin{array}{l}b_{\min }^{b} \\
{[\mathrm{mas}]}\end{array}$ & $\begin{array}{r}\mathrm{PA}^{b} \\
{\left[{ }^{\circ}\right]}\end{array}$ \\
\hline \multirow[t]{2}{*}{$2010-10-29$} & 8.4 & $\begin{array}{l}\text { PKS-AT-MP-HO- } \\
\text { CD-HH }\end{array}$ & - & $195 \pm 39$ & 0.079 & $294 \pm 59$ & 2.83 & 0.62 & -2.7 \\
\hline & & & 60 & $249 \pm 50$ & 0.064 & $301 \pm 60$ & 5.61 & 4.42 & -63 \\
\hline $1998-10-12$ & 1.5 & VLBA & - & $213 \pm 21$ & 0.41 & $605 \pm 61$ & 14.1 & 4.16 & -9.0 \\
\hline $1998-10-12$ & 1.5 & VLA & - & $726 \pm 73$ & 0.16 & $730 \pm 73$ & $1.15 \times 10^{4}$ & $3.0 \times 10^{3}$ & -1.7 \\
\hline
\end{tabular}

Notes. ${ }^{(a)}$ PKS: Parkes $(64 \mathrm{~m})$, AT: ATCA $(5 \times 22 \mathrm{~m})$, MP: Mopra $(22 \mathrm{~m})$, HO: Hobart $(26 \mathrm{~m})$, CD: Ceduna $(30 \mathrm{~m})$, HH: Hartebeesthoek $(26 \mathrm{~m})$; VLBA $8 \times 25 \mathrm{~m}$ (no Hancock and Brewster), including phased VLA antenna Y27; VLA in B configuration with $27 \times 25 \mathrm{~m}$ stations. ${ }^{(b)}$ Major and minor axis and position angle of the restoring beam.

\subsection{VLBI and VLA observations}

PKS 2004-447 was first observed in the framework of the TANAMI VLBI programme on 2010 Oct. 29. This observation was made at a frequency of $8.4 \mathrm{GHz}$. The TANAMI array consists of the five telescopes comprising the Australian Long Baseline Array (LBA), in combination with telescopes in Antarctica, Chile, and South Africa (see Table 1). The data were correlated on the DiFX software correlator (Deller et al. 2007, 2011) at Curtin University in Perth, Western Australia. The data were then calibrated and imaged following Ojha et al. (2010). And like them, we assume conservative uncertainties of the amplitude calibration for the TANAMI data of $20 \%$.

We also analysed the only other available archival VLBI observation obtained by the Very Long Baseline Array (VLBA) on 1998 Oct. 12 (project code: BD0050). We calibrated the data using the Astrophysical Image Processing System (AIPS, Greisen 2003). Hybrid imaging was conducted with the software package DIFMAP (Shepherd et al. 1994). We used uniform weighting to achieve the highest angular resolution. Lister \& Homan (2005) estimate an uncertainty of $5 \%$ for their $15 \mathrm{GHz}$ VLBA data. Given the very low declination of PKS 2004-447 for the VLBA and after repeating the imaging process several times, we assume a conservative uncertainty of $10 \%$ for the amplitude calibration of the VLBA data.

The Very Large Array (VLA) participated in "B" configuration in this VLBA observation. We extracted the VLA data from the archive and calibrated and imaged the data independently to check for missing flux in the VLBA image.

The parameters of the various images are given in Table 1. The noise level of the image $\sigma_{\text {rms }}$ was determined by fitting the noise-dominated pixels with a Gaussian distribution (Böck 2012) with the Interactive Spectral Interpretation System (ISIS) package (Houck \& Denicola 2000).

\section{Results}

\subsection{ATCA monitoring}

Figure 1 shows the ATCA light curve between $5.5 \mathrm{GHz}$ and $40 \mathrm{GHz}$ for the period 2010 Feb. 13 through 2014 Mar. 26. Figure 2 depicts the corresponding radio spectrum supplemented by individual ATCA calibrator database measurements at $1.7 \mathrm{GHz}, 2.1 \mathrm{GHz}, 33.0 \mathrm{GHz}, 35.0 \mathrm{GHz}, 43.0 \mathrm{GHz}$, and 45.0 GHz. This marks the first time that several simultaneous multi-frequency radio observations have been obtained for PKS 2004-447.

The data show only moderate variability in flux density up to a factor of two and a persistent steep radio spectrum over four years (see Table 2). There are two possible states of increased



Fig. 1. ATCA radio light curve at $5.5 \mathrm{GHz}$ (triangles), $9.0 \mathrm{GHz}$ (rectangles), 17.0 GHz (diamonds), 19.0 GHz (crosses), 38.0 GHz (asterisks), and $40 \mathrm{GHz}$ (circles) using only frequencies with more than two data points (see Table A.1).

activity. First, the $38 / 40 \mathrm{GHz}$ data indicate a possible weak flare on 2011 Nov. 08 without any obvious counterparts at lower frequencies. Second, the flux density increased at $5.5 \mathrm{GHz}$ and 9.0 GHz on 2014 Mar. 26. The relatively sparse sampling makes it difficult to study the evolution of the light curve in detail.

We adopt the variability index $V_{v}$ at frequency $v$ (Hovatta et al. 2008) as a basic quantity for the strength of the flaring activity,

$V_{v}=\frac{\left(S_{v, \text { max }}-\sigma_{v, \text { max }}\right)-\left(S_{v, \text { min }}+\sigma_{v, \text { min }}\right)}{\left(S_{v, \text { max }}-\sigma_{v, \text { max }}\right)+\left(S_{v, \text { min }}+\sigma_{v, \text { min }}\right)}$

where $S_{v, \max }$ and $S_{v, \text { min }}$ are the maximum and minimum flux density at frequency $v$ and as a basic indicator $\sigma_{v \text {,max }}$ and $\sigma_{v, \text { min }}$ the corresponding uncertainties. More sophisticated variability quantities would require better sampling (see, e.g., Richards et al. 2011 for an overview). We calculate $V_{v}$ between $5.5 \mathrm{GHz}$ and $19.0 \mathrm{GHz}$, which are the frequencies with the best coverage. The results indicate similar moderate variability between 0.22 and 0.34 at all four frequencies.

We investigate the simultaneous ATCA spectra by fitting a power law $S_{v}=k v^{\alpha}$ where $k$ is a proportionality constant and $\alpha$ the spectral index. We use a linear regression approach with $\log S_{v}=\log k+\alpha \log (v / \mathrm{GHz})$, where $v$ is in units of $\mathrm{GHz}$ to determine the spectral index and its uncertainty. Where possible, we produced fits for different spectral ranges, which are 




Fig. 2. Spectrum of ATCA monitoring (Fig. 1) between $1.7 \mathrm{GHz}$ and $45 \mathrm{GHz}$ from 2010 Feb. 13 to 2014 Mar. 26. Data for which spectral index fits were performed, are shown in colour. Simultaneous archival data were taken from G06 (blue, right-oriented triangles). Non-simultaneous archival data from various catalogues are shown in grey: AT20G (asterisk, Murphy et al. 2010; Chhetri et al. 2013), CRATES (bottom-oriented triangles, Healey et al. 2007, PKS90CAT (right-oriented triangles, Wright \& Otrupcek 1990), PMN (circles, Griffith \& Wright 1994; Wright et al. 1994), SUMSS (stars, Mauch et al. 2013), and ALMA Cal (rectangles, Fomalont et al. 2014).

Table 2. Results of power-law fit to various spectral ranges of the ATCA data $\left(S_{v}=k v^{\alpha}\right)$.

\begin{tabular}{lccc}
\hline \hline Date & $\alpha_{5.5-40}{ }^{a}$ & $\alpha_{5.5-19}{ }^{b}$ & $\alpha_{17-40}{ }^{c}$ \\
\hline $2004-04-12^{d}$ & & $-0.56 \pm 0.03$ & \\
\hline $2011-05-17$ & & $-0.72 \pm 0.05$ & \\
$2011-10-14$ & & $-0.75 \pm 0.05$ & \\
$2011-11-08$ & $-0.57 \pm 0.03$ & $-0.71 \pm 0.05$ & $-0.36 \pm 0.09$ \\
$2011-11-27$ & & $-0.56 \pm 0.04$ & \\
$2012-05-28$ & & & $-0.61 \pm 0.12$ \\
$2012-06-13$ & $-0.86 \pm 0.04$ & $-0.86 \pm 0.06$ & $-0.82 \pm 0.13$ \\
$2014-03-26$ & & $-0.62 \pm 0.04$ & \\
\hline
\end{tabular}

Notes. ${ }^{(a)}$ Parameters of fit to data between $5.5 \mathrm{GHz}$ and $40 \mathrm{GHz}$. (b) Parameters of fit to data between $4.8 \mathrm{GHz}$ and $19 \mathrm{GHz}$, i.e., for G06 data: 4.8-18 GHz, for our ATCA data: 5.5-19 GHz. ${ }^{(c)}$ Parameters of fit to data between $17 \mathrm{GHz}$ and $40 \mathrm{GHz} .{ }^{(d)}$ Data taken from G06.

listed in Table 2. Above 5.0 GHz, the spectral index varies moderately between $\sim-0.5$ and -0.9 . We have inspected the ratio of the data and the model and find that a power-law fit is sufficient to describe the analysed spectra. Only in the case of the data from 2011 Nov. 8, the power-law fit between $5.5 \mathrm{GHz}$ and $40 \mathrm{GHz}$ only marginally fits well due to a flattening of the spectrum above $17 \mathrm{GHz}$. (see also Sect. 4.1.)

\subsection{TANAMI VLBI image}

We present the first TANAMI $8.4 \mathrm{GHz}$ VLBI image of PKS 2004-447 in Fig. 3. The brightest feature, which we identify as the VLBI core, is located at the origin of the map. A one-sided jet extends in the north-west direction from the VLBI core. While the jet is partially resolved at full-array resolution beyond 10 mas from the centre, the tapered image in the bottom panel of Fig. 3 shows a continuous jet out to $\sim 30$ mas and a more diffuse region further downstream with an enhanced emission
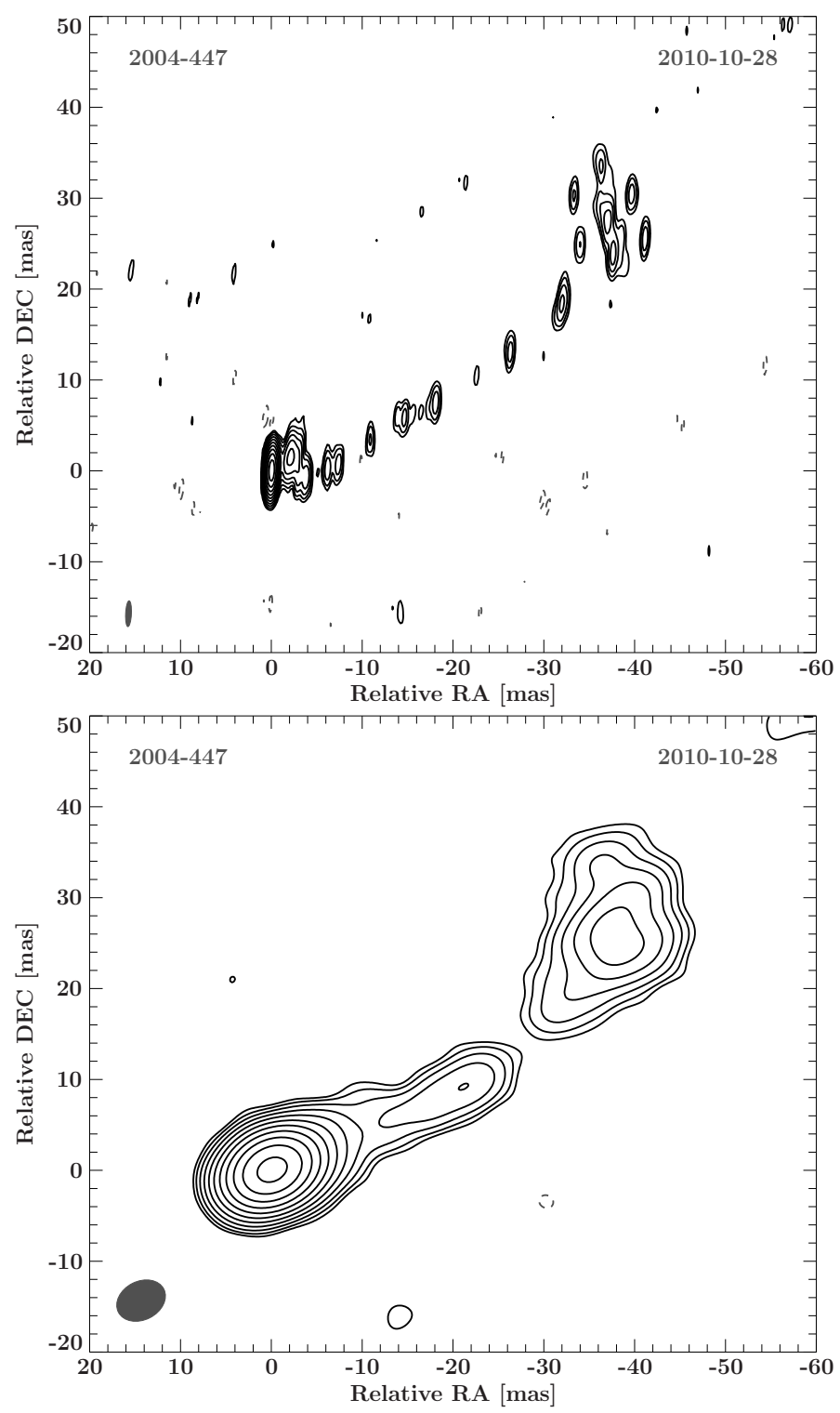

Fig. 3. Top: full-resolution TANAMI image of 2004-447 at $8.4 \mathrm{GHz}$; bottom: tapered TANAMI image of $2004-447$ at $8.4 \mathrm{GHz}$. The contour lines of each image start at three times the rms of the image, increasing logarithmically by a factor of two. Negative flux density noise peaks are shown by dashed grey contour lines. The grey ellipse in the bottom left of both images depicts their respective restoring beams. The image parameters are shown in Table 1.

region or "hot spot" at $\sim 45$ mas. This image was obtained by applying a Gaussian taper with a strength of 0.1 at $60 \mathrm{M} \lambda$ to the visibility data and by repeating the full hybrid imaging process. The untapered TANAMI image contains a total flux density of (294 \pm 59$) \mathrm{mJy}$. The dynamic range DR of the image, i.e., the ratio between the peak flux density and five times the rms noise, is $D R_{8.4 \mathrm{GHz}} \approx 490$.

To further characterize the core, we deleted CLEAN components contained within 1 mas of the core and fit an elliptical Gaussian component to the core, using ISIS (Houck \& Denicola 2000) with a direct link to DIFMAP (Großberger 2014). This enabled us to calculate the given $1 \sigma$-uncertainties using $\chi^{2}$-statistics. The fit parameters are given in Table 3 . The compact core component yields a flux density of $\left(229.8_{-0.2}^{+0.4} \pm\right.$ $35_{\text {sys }}$ ) mJy, which is almost $80 \%$ of the total flux density. We 
R. Schulz et al.: The gamma-ray emitting radio-loud narrow-line Seyfert 1 galaxy PKS 2004-447. II.

Table 3. Parameters of the elliptical Gaussian component fit to the central feature of the TANAMI and VLBA images.

\begin{tabular}{cccccc}
\hline \hline $\begin{array}{c}v \\
{[\mathrm{GHz}]}\end{array}$ & $\begin{array}{c}S_{\mathrm{c}} \\
{[\mathrm{mJy}]}\end{array}$ & $\begin{array}{c}a_{\text {maj }}{ }^{a} \\
{[\mathrm{mas}]}\end{array}$ & $\begin{array}{c}\mathrm{Ratio}^{a} \\
{[\mathrm{mas}]}\end{array}$ & $\begin{array}{c}\theta_{\mathrm{PA}}{ }^{a} \\
{\left[{ }^{\circ}\right]}\end{array}$ & $\begin{array}{c}T_{\mathrm{b}}{ }^{b} \\
{\left[10^{10} \mathrm{~K}\right]}\end{array}$ \\
\hline 8.4 & $229.8_{-0.2_{\text {stat }}^{+0.4} \pm 35_{\text {sys }}}$ & $0.3651 \pm 0.0016$ & $0.64_{-0.14}^{+0.15}$ & $88 \pm 3$ & $5.7 \pm 1.4$ \\
1.5 & $248.8 \pm 0.4_{\text {stat }} \pm 25_{\text {sys }}$ & $2.93_{-0.12}^{+0.14}$ & $0.63 \pm 0.04$ & $31_{-7}^{+8}$ & $3.4 \pm 0.3$ \\
\hline
\end{tabular}

Notes. Errors are given in $1 \sigma$ interval. ${ }^{(a)}$ Major axis, ratio of major and minor axes, and position angle of the component. ${ }^{(b)}$ The highest uncertainty of $S_{\mathrm{c}}, a_{\text {maj }}$ and the axis ratio was taken to calculate the error in the brightness temperature.

calculated the brightness temperature $T_{\mathrm{b}}$ following e.g., Kovalev et al. $(2005)$ to be $(5.7 \pm 1.4) \times 10^{10} \mathrm{~K}$. The $1 \sigma$ error is predominantly determined by the uncertainty of the axis ratio of the component. The brightness temperature is well below the inverse-Compton limit of $10^{12} \mathrm{~K}$ (Kellermann \& Pauliny-Toth $1969)$ and is consistent with the equipartition limit of $\sim 10^{11} \mathrm{~K}$ (Readhead 1994). Additionally, we fitted a circular Gaussian distribution to the "hot spot" by only deleting the CLEAN components within this region and measured a brightness temperature of about $10^{7} \mathrm{~K}$.

\subsection{VLBA and VLA image}

Figure 4 shows PKS 2004-447 at $1.5 \mathrm{GHz}$ based on the 1998 Oct. 12 VLBA observation. We find a total flux density in the image of $(605 \pm 61) \mathrm{mJy}$ and a dynamic range of $D R_{1.5 \mathrm{GHz}} \approx 100$, which is a comparatively low level owing to the very low declination of the source for the VLBA and the resulting sparse distribution of visibilities in the $(u, v)$-plane. The radio morphology shows a bright central core with a jet extending to the north-west and substantial diffuse emission on the eastern side with a total extent of about 140 mas. The "hot spot" at $\sim 45$ mas of the core shows a brightness temperature of $\sim 10^{7} \mathrm{~K}$ at $1.5 \mathrm{GHz}$.

Following Sect. 3.2 we fitted an elliptical component to the core, accounting for CLEAN-components within 5 mas of the core. The resulting component has a flux density that contains roughly $40 \%$ of the total flux density (see Table 3 ). We derive a core brightness temperature of $(3.4 \pm 0.3) \times 10^{10} \mathrm{~K}$ at $1.5 \mathrm{GHz}$.

The VLA image in Fig. 4 shows that PKS 2004-447 is compact at $1.5 \mathrm{GHz}$ for the VLA in B configuration. We fitted an elliptical Gaussian component to the visibility data, which results in an unresolved component sufficient to describe the visibility data without significant residuals. No extended emission is detected using a detection limit of three times the noise level $(0.48 \mathrm{mJy} /$ beam $)$. As a result, the beam of the VLA observation limits the projected linear size to less than $11 \mathrm{kpc}$. The total flux density in the VLBA and VLA image is consistent considering the uncertainties in amplitude calibration. The value of the total flux density in the VLBA and VLA images indicates that $\sim 80 \%$ of the source flux density is located within $\sim 140$ mas, corresponding to $\sim 530 \mathrm{pc}$ in the east-west direction, i.e., in the direction of the minor axis of the VLA beam.

\section{Discussion}

\subsection{Discussion of the radio spectrum}

Our ATCA monitoring shows that PKS 2004-447 exhibits a persistent steep radio spectrum above $5 \mathrm{GHz}$ over four years of monitoring. This is consistent with earlier snapshot radiospectral observations in 2001 and 2004: Osh01 reported a spectral index between $2.7 \mathrm{GHz}$ and $5.0 \mathrm{GHz}$ of $\alpha_{2.7-5}=-0.67$ from a power-law fit to simultaneous ATCA observations. G06 conducted ATCA observations between $1.4 \mathrm{GHz}$ and $17.9 \mathrm{GHz}$ on 2004 Apr. 12/13, deriving a spectral index of $\alpha_{1.4-18}=-0.52$.

To compare changes in the spectra from our ATCA TANAMI monitoring and G06, we selected an overlapping spectral range, i.e., between $4.8 \mathrm{GHz}$ and $19 \mathrm{GHz}$. We fitted these data points as described in Sect. 3.1. The results are listed in Table 2. The data show moderate spectral variability with the spectral slope varying between $\sim-0.5$ and $\sim-0.9$ with a weighted mean of $\alpha_{\mathrm{wm}}=-0.68 \pm 0.10$ and a median of $\alpha_{\text {median }} \approx-0.72$. The mean spectral index is consistent with Osh01.

A possible flattening above $17 \mathrm{GHz}$ is observed in the epoch 2011 Nov. 8. This is due to the aforementioned increased flux density at $38 \mathrm{GHz}$ and $40 \mathrm{GHz}$. Intriguingly, the X-ray light curve (presented in Paper I) shows a high activity state on 2011 Sept. 17. Additionally, we find the X-ray spectra of PKS 2004-447 in Paper I to be well accounted for by a single non-thermal power law, possibly from the jet. However, the relatively sparse sampling makes it difficult to draw strong conclusions regarding a possible correlation.

In previous studies, PKS 2004-447 has been assigned a flatter spectral index $(\alpha>-0.5)$ at low frequencies $(<5 \mathrm{GHz})$ based on non-simultaneous data (e.g., Osh01, Healey et al. 2007). We calculated the simultaneous spectral index between $1.38 \mathrm{GHz}$ and $2.4 \mathrm{GHz}$ from G06 to be $\alpha_{1.38-2.4}=-0.21 \pm 0.08$, which is significantly flatter than above $2.4 \mathrm{GHz}(\alpha=-0.56 \pm 0.03)$. This suggests that a turnover in the radio spectrum occurs below $2 \mathrm{GHz}$ as is observed for CSS sources (O'Dea 1998).

The weighted average spectral luminosity at $5 \mathrm{GHz}$ is calculated by extrapolating the flux density of our ATCA data at $5.5 \mathrm{GHz}$. We use the fitted spectral index for the $K$ correction following Ghisellini et al. (2009), for example. This yields $L_{5, \mathrm{wm}}=(7.4 \pm 1.1) \times 10^{25} \mathrm{~W} \mathrm{~Hz}^{-1}$, which is consistent with the lower end of the $5 \mathrm{GHz}$ luminosity distribution in the CSS/GPS sample discussed by O'Dea (1998). The combination of archival and our new flux density measurements supports the classification of PKS 2004-447 as a CSS source, as originally suggested by Osh01.

\subsection{Parsec-scale structure and jet orientation}

The $1.5 \mathrm{GHz}$ VLBA image shows a bright central component and a jet towards the north-west and substantial diffuse emission towards the east. The central and western structure is consistent with an image based on the same data by Orienti et al. (2015), in which the diffuse eastern emission, however, is not visible because of the smaller field of view.

We measure high brightness temperatures of $>10^{10} \mathrm{~K}$ in the VLBI core at both frequencies. The non-simultaneous spectral emission of the core is relatively flat with a spectral index of $\alpha_{\mathrm{VLBI}, 1.5-8.4} \sim-0.04$. A core-jet structure has been observed in 



Fig. 4. VLBA (top) and VLA (bottom) image of $2004-447$ at $1.5 \mathrm{GHz}$ with the image parameters given in Table 1 . Contour lines begin at 3 times the image rms and increase logarithmically by a factor of 2 . Grey dashed contour lines indicate negative flux density. The grey ellipse in the bottom left corner depicts the restoring beam.

some CSS sources but is more common in GPS sources (e.g., O'Dea 1998; Fanti et al. 2001 and references therein).

The angle of the jet to the line of sight $\theta$ can be estimated using the jet-to-counterjet ratio $R$, the intrinsic jet velocity $\beta$, and the spectral index $\alpha$ via

$R=\frac{S_{\mathrm{J}}}{S_{\mathrm{CJ}}}=\left(\frac{1+\beta \cos \theta}{1-\beta \cos \theta}\right)^{2-\alpha}$

where $S_{\mathrm{J}}$ and $S_{\mathrm{CJ}}$ are the jet and counter-jet flux densities, respectively (e.g., Urry \& Padovani 1995). Assuming that the diffuse emission on the eastern side of the core at $1.5 \mathrm{GHz}$ can be attributed to the counter jet yields $S_{\mathrm{CJ}} \approx 59 \mathrm{mJy}$. Excluding the core region results in an integrated flux density in the jet of $S_{\mathrm{J}} \approx 313 \mathrm{mJy}$. This leads to an upper limit of $\theta_{\mathrm{LOS}, 1.5} \lesssim 74^{\circ}$ for an optically thin jet with spectral index of $\sim-1$ (Hovatta et al. 2014) and $\beta \rightarrow 1$. Since the counter jet is not detected at $8.4 \mathrm{GHz}$, we use the peak flux density per beam in the first feature of the jet next to the core $\left(S_{\mathrm{J}} \approx 9.5 \mathrm{mJy}\right.$ beam $\left.^{-1}\right)$ and five times the noise level on the side of the counter jet as a detection limit $\left(S_{\mathrm{CJ}} \approx 0.1 \mathrm{mJy}\right.$ beam $\left.^{-1}\right)$. This yields a lower upper limit for the jet angle to the line of sight of $\theta_{\mathrm{LOS}, 8.4} \lesssim 50^{\circ}$. Conversely, the jet-to-counter-jet ratios provide a lower limit on the intrinsic jet velocity with $\beta_{1.5}>0.27$ and $\beta_{8.4}>0.67$ at $1.5 \mathrm{GHz}$ and $8.4 \mathrm{GHz}$, respectively. Differences in the results between both frequencies can be perhaps attributed, at least in part, to different scales of the emission region used for the estimate of $S_{\mathrm{J}}$ and $S_{\mathrm{CJ}}$. This provides an independent proof of relativistic bulk motion in PKS 2004-447.

The coherent emission at $\sim 45$ mas may indicate a moderately pronounced "hotspot" with a brightness temperature of $10^{7} \mathrm{~K}$ at $1.5 \mathrm{GHz}$. Such brightness temperatures have also been measured in "hot spots" of CSS/GPS sources (Dallacasa et al. 2002a,b). Diffuse emission north of the "hotspot" can be seen in both images. The offset in position angle may indicate a backflow of jet plasma after an interaction with the surrounding medium. The eastern diffuse emission is not detected in our TANAMI image at $8.4 \mathrm{GHz}$. Given the non-detection on a $5 \sigma_{\text {rms }}$ level, this indicates a spectral index steeper than -1.9. The full extent of the radio emission from PKS 2004-447 at $1.5 \mathrm{GHz}$ picked up by the VLBA is $\sim 140$ mas, which corresponds to a projected linear size of roughly $530 \mathrm{pc}$.

The linear size of PKS 2004-447 can also be assessed indirectly from the radio spectrum. O'Dea \& Baum (1997) studied the relationship between rest turnover frequency $v_{\mathrm{m}}$ and $l_{\mathrm{ps}}$ of a sample of CSS and GPS sources, finding an anti-correlation $v_{\mathrm{m}} \propto l_{\mathrm{ps}}^{-0.65}$. Since the turnover in the radio spectrum is only partially visible below $2 \mathrm{GHz}$, we adopted the lowest frequency measurement of the PKS90CAT data as an estimate for the turnover frequency with $v_{\mathrm{m}, \mathrm{obs}}=408 \mathrm{MHz}$ and $v_{\mathrm{m}} \sim 500 \mathrm{MHz}$ in the observer and source rest frames, respectively. This yields an estimate of the linear size from the spectrum of $l_{\mathrm{ps}} \sim 2 \mathrm{kpc}$, which probably overestimates the true size, but still consistent with a CSS source.

Another estimate of $l_{\mathrm{ps}}$ can be obtained from the ATCA Calibrator Database data at $4 \mathrm{~cm}$. Here, PKS 2004-447 shows a flat visibility data distribution (out to $d_{\max } \approx 150 \mathrm{k} \lambda$ ) in the $(u, v)$-plane and no significant defect, which suggests that PKS 2004-447 remains unresolved. Using $\sin \phi \approx 1.22 \lambda / d_{\max }$, this yields an angular size of $\phi \lesssim 11^{\prime \prime} 7$ to a linear projected size of only $l_{\mathrm{ps}} \lesssim 6.4 \mathrm{kpc}$, corresponding to $v_{\mathrm{m}} \gtrsim 240 \mathrm{MHz}$ and $v_{\mathrm{m}, \mathrm{obs}} \gtrsim 200 \mathrm{MHz}$ for the turnover frequency in the source and observer frames, respectively.

The various estimates of the linear size and the turnover frequencies of PKS 2004-447 are all consistent with CSS sources. Nevertheless, we adopt the directly measured limit of $l_{\mathrm{ps}} \lesssim$ $11 \mathrm{kpc}$ from the VLA image as a conservative upper limit on the linear size. Recently, Richards \& Lister (2015) have detected extended emission in three RL-NLS1s using observations with the VLA at a frequency of $9.0 \mathrm{GHz}$. The study achieved sub-arcsec resolution and high sensitivity between $0.021 \mathrm{mJy} /$ beam and $0.05 \mathrm{mJy} /$ beam. The authors suggest that extended emission will be detected in more sources if higher sensitivity observations are conducted. The size of the extended emission in these sources is larger than the beam of our VLA observation at $1.5 \mathrm{GHz}$, indicating that extended emission may not have been detected due to the sensitivity. To analyse this further, we extrapolated the 
R. Schulz et al.: The gamma-ray emitting radio-loud narrow-line Seyfert 1 galaxy PKS 2004-447. II.

Table 4. Radio properties of $\gamma$-NLS1.

\begin{tabular}{|c|c|c|c|c|c|c|c|c|c|c|}
\hline Source & $z^{a}$ & $\begin{array}{c}T_{\mathrm{b}, 15}{ }^{b} \\
{\left[10^{11} \mathrm{~K}\right]}\end{array}$ & $\begin{array}{c}T_{\mathrm{b}, 8}{ }^{c} \\
{\left[10^{11} \mathrm{~K}\right]}\end{array}$ & $\begin{array}{c}l_{\mathrm{ps}}{ }^{d} \\
{[\mathrm{kpc}]} \\
\end{array}$ & Radio spectrum $^{e}$ & Variability $^{f}$ & $V_{4.85 / 5.5^{g}}$ & $V_{8.35 / 9.0^{g}}$ & $V_{\mathrm{X}}^{h}$ & $\mathrm{RL}_{1.4}^{i}$ \\
\hline $1 \mathrm{H} 0323+342$ & 0.061 & $\begin{array}{c}7.3 \pm 2.7 \\
(6.2)\end{array}$ & - & 24 & steep, flat & $\mathrm{F} / \mathrm{S}$ & 0.30 & 0.32 & 0.37 & 318 \\
\hline SBS $0846+513$ & 0.584 & $\begin{array}{c}36 \pm 27 \\
(7.5)\end{array}$ & - & $<2.2$ & flat, inverted & $\mathrm{F} / \mathrm{S}$ & 0.13 & 0.14 & 0.42 & 4496 \\
\hline PMN J0948+0022 & 0.585 & $\begin{array}{c}64 \pm 15 \\
(44)\end{array}$ & $\begin{array}{c}4.0 \pm 1.3 \\
(1.7)\end{array}$ & 104 & flat, inverted & $\mathrm{F} / \mathrm{S}$ & 0.55 & 0.65 & 0.50 & 846 \\
\hline [HB89] 1219+044 & 0.966 & $\begin{array}{c}119 \pm 95 \\
(96)\end{array}$ & 43 & $\sim 80$ & flat & $\mathrm{F} /-$ & - & - & - & 1700 \\
\hline PKS $1502+036$ & 0.409 & $\begin{array}{c}3.9 \pm 1.1 \\
(3.0)\end{array}$ & - & $<25$ & GHz-peaked, flat & $\mathrm{F} / \mathrm{S}$ & 0.27 & 0.30 & -0.4 & 3364 \\
\hline FBQS J1644+2619 & 0.145 & - & 6.3 & 60 & - & - & - & - & - & 396 \\
\hline PKS 2004-447 & 0.24 & - & $0.4 \pm 0.1$ & $<11$ & CSS-like, steep & $\mathrm{F} / \mathrm{S}$ & 0.23 & 0.32 & 0.45 & 6358 \\
\hline
\end{tabular}

Notes. Hyphens indicate that no data were available. ${ }^{(a)}$ Redshift from NED. ${ }^{(b)}$ Mean brightness temperature $T_{\mathrm{b}}$ at $15 \mathrm{GHz}$ based on (1) except for [HB89] 1219+044 (2), brackets refer to the median. ${ }^{(c)} T_{\mathrm{b}}$ at $\sim 8 \mathrm{GHz}$, mean $T_{\mathrm{b}}$ based on (1) and the median in brackets for PMN J0948+0022; 1H 0323+342 from (3), FBQS J1644+2619 from (4), [HB89] 1219+044 from (5). ${ }^{(d)}$ Projected linear size from VLA observations; for SBS $0846+513$ and PKS 1502+036 the major beam axis of the highest resolution VLA observation from (6) and (7), respectively, was used as a conservative upper limit; Reference (4) for 1H 0323+342, PMN J0948+0022, FBQS J1644+2619; Reference (8) for [HB89] $1219+044$. (e) References for 1H0323+342 (3, 9), SBS 0846+513 (6, 9, 10), PMN J0948+0022 (11, 9), PKS 1502+036 (7, 9), and FBQS J1644+2619 (4). (f) F: flux variability; S: spectral variability from simultaneous data. ${ }^{(g)}$ Variability index for $1 \mathrm{H} 0323+342$, SBS $0846+513$, PMN J0948+0022, and PKS $1502+036$ at 4.85 and $8.35 \mathrm{GHz}$, respectively, from (9); PKS 2004-447 at 5.5 and $9.0 \mathrm{GHz}$. ${ }^{(h)}$ Variability index at X-ray energies from $0.3-10 \mathrm{keV}$ for $1 \mathrm{H} 0323+342$ (1), SBS 0846+513 (10), PMN J0948+0022 (1), and PKS 1502+036 (7); 0.5-10 keV for PKS 2004-447 (12); For PKS $1502+036$ all flux measurements were consistent within the given uncertainties, yielding a negative value. ${ }^{(i)}$ Reference (13) for all except [HB89] 1219+044 (14).

References. (1) Foschini et al. (2015); (2) Lister et al. (2013); (3) Wajima et al. (2014); (4) Doi et al. (2012); (5) Pushkarev \& Kovalev (2012); (6) D'Ammando et al. (2012); (7) D'Ammando et al. (2013a); (8) Kharb et al. (2010); (9) Angelakis et al. (2015); (10) D'Ammando et al. (2013b); (11) D’Ammando et al. (2014); (12) Paper I; (13) Foschini (2011); (14) Yao et al. (2015).

detection limit of our VLA observation at $1.5 \mathrm{GHz}$, i.e. three times the noise level $(0.48 \mathrm{mJy} /$ beam $)$ to $9.0 \mathrm{GHz}$ using a spectral index of -0.7 for synchrotron emission. This yields a flux density of $\sim 0.14 \mathrm{mJy} /$ beam corresponding to 14 times the lowest sensitivity in Richards \& Lister (2015). However, our ATCA data at $9.0 \mathrm{GHz}$ does not indicate extended emission. At this frequency, the resolution of ATCA is about 1.7 , and the sensitivity of this observation is approximately $\sim 0.07 \mathrm{mJy} /$ beam, so comparable to Richards \& Lister (2015). As a result, we cannot completely rule out the existence of very faint extended emission on scales larger than $11 \mathrm{kpc}$ (VLA limit) or $6.4 \mathrm{kpc}$ (ATCA limit), but it would most likely require observations at even higher sensitivity than in Richards \& Lister (2015). We therefore consider $l_{\mathrm{ps}} \lesssim 11 \mathrm{kpc}$ as a reasonable upper limit. Applying the $v_{\mathrm{m}}-l_{\mathrm{ps}^{-}}$ relationship yields $v_{\mathrm{m}, \mathrm{obs}} \gtrsim 140 \mathrm{MHz}$, which is currently not accessible by our radio data.

In a recent study Kunert-Bajraszewska et al. (2010) have compared the $1.4 \mathrm{GHz}$ luminosity with the linear size and redshift of a sample of CSS, GPS and low-luminous compact (LLC) objects. Using the flux density measured by G06 at $1.38 \mathrm{GHz}$ and $\alpha_{1.38-2.4}$ from Sect. 4.1 yields a luminosity of $L_{1.38} \approx 1.2 \times$ $10^{26} \mathrm{~W} \mathrm{~Hz}^{-1}$. This puts PKS 2004-447 at the lower end of the luminosity distribution in the combined CSS sample of KunertBajraszewska et al. (2010). The linear size of PKS 2004-447 agrees well with the CSS sample when considering even the lowest possible estimate of the linear size from the VLBA image. In addition, PKS 2004-447 also fits with the distribution of LLC sources, where it is at the upper end of the luminosity distribution. This is to be expected because one of the selection criteria of the LLC sample is $L_{1.4} \leq 10^{26} \mathrm{~W} \mathrm{~Hz}^{-1}$. The distribution of $L_{1.4}$ with redshift gives a similar result.

Our study confirms the CSS-like nature of PKS 2004-447 suggested by G06. As such, PKS 2004-447 belongs to an even more elusive class of $\gamma$-ray detected AGNs than $\gamma$-NLS 1 according to the 3LAC (Ackermann et al. 2015). Only one other CSS source has been associated with a $\gamma$-ray counterpart in the $3 \mathrm{LAC}$.

While CSS/GPS sources have been discussed in the context of young radio sources or frustrated AGNs (e.g., O'Dea 1998; Stanghellini 2003; Kunert-Bajraszewska et al. 2010 and references therein), more recent publications favour the paradigm of young AGNs over the older radio-loud population (e.g., Fanti et al. 2011; Randall et al. 2011). Both paradigms are also discussed for $\gamma$-NLS1(e.g., Abdo et al. 2009c; Foschini et al. 2015; Berton et al. 2015), and it has been proposed that CSS and RL-NLS1 are linked (e.g., Komossa et al. 2006; Doi et al. 2012; Caccianiga et al. 2014; Komossa et al. 2015; Gu et al. 2015). Recently, Caccianiga et al. (2015) have found evidence of significant star formation activity in a sample of RL-NLS1s, favouring the scenario that RL-NLS1s are young AGNs. A similar case is the recently reported $\gamma$-ray loud AGN PMN J1603-4904, which also shows a moderately steep radio spectrum (Müller et al. 2014). It is compact on arcsec scales and highly symmetric on mas scales, which is reminiscent of a compact symmetric object that is also considered to be young radio sources (e.g., Wilkinson et al. 1994; An et al. 2012).

\subsection{Comparison with other $\gamma-N L S 1 s$}

Among the small sample of $\gamma$-NLS1s (see Foschini et al. 2015; D'Ammando et al. 2015; Yao et al. 2015), the sources besides PKS 2004-447, which were studied best so far in the radio regime and which yielded a significant ${ }^{5} \gamma$-ray detection by Fermi/LAT, are listed in Table 4. PKS 2004-447 is the only

$5 \quad T S \geq 25$, see Foschini et al. (2015), D'Ammando et al. (2015), Yao et al. (2015). 
$\gamma$-NLS1 detected that is located in the southern hemisphere, and it is the radio loudest of them. In this section, we compare our results for PKS 2004-447 with these other $\gamma$-NLS1s. A comparison of the X-ray properties is presented in Paper I.

\subsubsection{Flux density variability and spectrum}

In Sect. 3 we showed that PKS 2004-447 exhibits radio flux density variability up to a factor of 2 on time scales of months to years at centimetre wavelengths, which are comparable to values reported for other $\gamma$-NLS1s (e.g., Zhou et al. 2007; D'Ammando et al. 2013a,b; Angelakis et al. 2015). The recent studies by Foschini et al. (2015) and Angelakis et al. (2015) have presented the densest, simultaneous multi-frequency radio monitoring of four $\gamma$-NLS1s. The strength of the flaring activity differs for each source and depends strongly on frequency with a frequency delay of the flares, as previously known for blazars. To compare the variability index $V_{v}$ calculated for PKS $2004-447$ at $5.5 \mathrm{GHz}$ and 9.0 GHz in Sect. 3.1, we calculate $V_{v}$ for the $\gamma$-NLS1s discussed by Angelakis et al. (2015) and list the results for $\sim 5 \mathrm{GHz}$ and $\sim 8.4 \mathrm{GHz}$ in Table 4. PMN J0948+0022 has the highest variability index of the five sources, which is also the case at other frequencies except for $23 \mathrm{GHz}$ and $32 \mathrm{GHz}$, which is dominated by $1 \mathrm{H} 0323+342$, despite the lower sampling of $1 \mathrm{H} 0323+342$. SBS $0846+513$ has the lowest cadence of the four sources in Angelakis et al. (2015), though similar to PKS 2004-447. A comparison with the distribution of $V_{v}$ at the two frequencies of the sample in Hovatta et al. (2008), which consisted mostly of blazars, showed that the $\gamma$-NLS1s are located at the lowest end of the distribution except for PMN J0948+0022, which is consistent with the median values for quasars and BL Lacs.

The study by Angelakis et al. (2015) revealed strong differences in the simultaneous radio spectra that resemble types of radio spectra found in blazars by the F-GAMMA programme (see Angelakis et al. 2012), supporting Yuan et al. (2008) regarding the link between RL-NLS1 and blazars using simultaneous data. However, a CSS-like spectrum of PKS 2004-447, with a possible turnover in the $\mathrm{MHz}$ range, does not fit the classification by Angelakis et al. (2012) and is unique among all $\gamma$-NLS1s. Berton et al. (2015) compared the population of flat-spectrum NLS1s from Foschini et al. (2015), which includes all $\gamma$-NLS1s with a sample of steep-spectrum NLS1s. Based on the KolmogorovSmirnov test, their findings suggest that both samples may stem from the same population. Foschini et al. (2015) interpret this in such a way that steep-spectrum NLS1s have a larger angle to the line of sight than flat-spectrum NLS1s. These characteristics fit PKS 2004-447 with the difference that no steep-spectum NLS1 has been detected in the $\gamma$-ray regime so far. The single exception may be RX J2314.9+2243, for which the $\gamma$-ray detection is only tentative (Komossa et al. 2015 and references therein).

The variety in the radio spectra of $\gamma$-NLS1s is not matched in the X-rays (see Paper I and Foschini et al. 2015), but differences seem to exist in the flaring activity of these sources. For this purpose, we calculated the variability index based on X-ray data $V_{\mathrm{X}}$ from D'Ammando et al. (2013a,b) and Foschini et al. (2015) for the northern sources and Paper I for PKS 2004-447. The amount of available data strongly varies between the four sources. PKS 1502+036 has the lowest amount of data, which covers the shortest time range. All of its flux measurements were consistent within the uncertainties. For 1H 0323+342, PMN J0948+0022, and PKS 2004-447 the sampling is sufficient for comparison with $V_{v}$. Again, PMN J0948+0022 yields the highest value, but SBS 0846+513 and PKS 2004-447 show $V_{\mathrm{X}}>V_{v}$, suggesting stronger variability at $\mathrm{X}$-ray energies. The photon index of the
X-ray spectra of the $\gamma$-NLS1s lies between 1.5 and 2.0 (Paper I), which fits into the range covered by BLLac objects (Foschini et al. 2015). However, the comparison of the $\gamma$-ray and X-ray luminosity by Foschini et al. (2015) indicates a distribution similar to flat-spectrum radio quasars.

\subsubsection{Radio morphology and brightness temperature}

Our deep VLBI observation of PKS 2004-447 confirms that on parsec scales, all considered $\gamma$-NLS1s possess a one-sided jet that extends from a dominant central core (e.g., Doi et al. 2006; D'Ammando et al. 2013a,b; Wajima et al. 2014). This morphology is reminiscent of blazars (e.g., Lister et al. 2013 and references therein). In contrast to this, the kilo-parsec scale, projected linear size is usually smaller than for radio-loud AGNs (e.g., Yuan et al. 2008; Doi et al. 2012 and references therein) and the extent of the large scale structure has been measured directly for only four sources (see Table 4).

The one-sidedness of the radio jet is a strong indication of relativistic beaming, which often gives rise to apparent superluminal motion of distinct jet features (see, e.g., Lister et al. 2013). SBS $0846+513$ is the only source in this group, for which the apparent superluminal motion of $\beta_{\text {app }}=(9.3 \pm 0.6) c$ (D'Ammando et al. 2013b) has been reported so far. However, Lister et al. (2013) report a maximum apparent speed of $0.82 c$ for [HB89] 1219+044.

Another indicator of relativistic beaming is the brightness temperature determined from the VLBI core $T_{\mathrm{B}}$. We computed the mean $T_{\mathrm{B}}$-value at $15 \mathrm{GHz}$ and $\sim 8 \mathrm{GHz}$ for the northern $\gamma$-NLS1s based on available data from Doi et al. (2012), Lister et al. (2013), and Foschini et al. (2015). In the case of [HB89] 1219+044, the $T_{\mathrm{B}}$ value was taken directly from Pushkarev \& Kovalev (2012). We find $T_{\mathrm{B}}$ to be well in excess of the equipartition limit and in three cases above the inverseCompton limit. Such values are consistent with blazars (e.g., Kovalev et al. 2005). PKS 2004-447 shows the lowest $T_{\mathrm{B}}$ value in the sample, about two orders of magnitude below the highest observed value by [HB89] 1219+044. This suggests intrinsic differences in Doppler boosting of the jet emission, which is consistent with studies of the variability brightness temperature $T_{\mathrm{B}, \mathrm{var}}$ from radio light curves (e.g., Angelakis et al. 2015).

\section{Summary and conclusion}

We have presented the highest resolution VLBI image obtained so far for the $\gamma$-NLS1 galaxy PKS 2004-447. PKS 2004-447 is the only southern source in an elusive sample and is the radioloudest one. The image reveals a single-sided jet extending to the north-west from a dominant core with a brightness temperature of $(5.7 \pm 1.4) \times 10^{10} \mathrm{~K}$. The first long-term multi-frequency flux density measurements with ATCA between $5.5 \mathrm{GHz}$ and $40 \mathrm{GHz}$ demonstrates a persistent steep radio spectrum with a spectral index betweeen -0.5 and -0.9 and moderate flux density variability.

We compared our results within the small sample of $\gamma$-NLS1s. We find that the brightness temperatures of the VLBI cores are either close to or in excess of the equipartition limit ranging over two orders of magnitude. In combination with the single-sided jet morphology on parsec scales, this demonstrates the importance of relativistic beaming of the radio emission in all of these sources. In addition, the variability index at $\sim 5 \mathrm{GHz}$ and $\sim 8 \mathrm{GHz}$ shows significant differences in variability. The shape of the radio spectrum differs strongly among $\gamma$-NLS1s with the 
persistent CSS-like spectrum of PKS 2004-447 being a unique exemplar. It is also extremely rare among $\gamma$-ray loud AGN listed in the 3LAC.

In Paper I, we showed that the X-ray spectra of $\gamma$-NLS1s do not show as much diversity as the radio spectra. In this paper, we calculated the variability index at X-ray energies and found that PMN J0948+0022 has the highest variability among sources with sufficient data.

The TANAMI programme is continuing the VLBI observations and flux density measurements of PKS 2004-447 to study temporal changes in the jet and radio spectrum to constrain or measure the jet speed. An accurate measurement of the turnover frequency can come from observations below $400 \mathrm{MHz}$, making PKS 2004-447 an intriguing candidate for studies with highsensitivity, low-frequency precursors of the Square Kilometre Array (e.g., Wayth et al. 2015).

Acknowledgements. We thank the MPIfR internal referee S. Komossa and the anonymous journal referee for their insightful comments, which improved the manuscript in its final form. This work was funded by Deutsche Forschungsgemeinschaft grant WI 1860/10-1. We acknowledge support by the Deutsches Zentrum für Luft- und Raumfahrt under contract number 50 OR 1303, by the Spanish MINECO projects AYA2009-13036-C02-02 and AYA2012-38491-C02-01, and by the Generalitat Valenciana projects PROMETEO/2009/104 and PROMETEO/II/2014/057, as well as by the MP0905 action "Black Holes in a Violent Universe". This research was funded in part by NASA through Fermi Guest Investigator grants NNH09ZDA001N, NNH10ZDA001N, NNH12ZDA001N, and NNH13ZDA001N-FERMI (proposal numbers $31263,41213,61089$, and 71326 , respectively). This research was supported by an appointment to the NASA Postdoctoral Program at the Goddard Space Flight Center, administered by Oak Ridge Associated Universities through a contract with NASA. The Australian Long Baseline Array and the Australia Telescope Compact Array are part of the Australia Telescope National Facility, which is funded by the Commonwealth of Australia for operation as a National Facility managed by CSIRO. This research made use of the Interactive Spectral Interpretation System (ISIS) (Houck \& Denicola 2000). This research made use of a collection of ISIS scripts provided by the Dr. Karl Remeis observatory, Bamberg, Germany at http://www.sternwarte.uni-erlangen.de/ isis/. This research made use of the NASA/IPAC Extragalactic Database (NED), which is operated by the Jet Propulsion Laboratory, California Institute of Technology, under contract with the National Aeronautics and Space Administration. This research made use of the VizieR catalogue access tool, CDS, Strasbourg, France. This research made use of data from the MOJAVE database that is maintained by the MOJAVE team (Lister et al. 2009).

\section{References}

Abdo, A. A., Ackermann, M., Ajello, M., et al. 2009a, ApJ, 699, 976 Abdo, A. A., Ackermann, M., Ajello, M., et al. 2009b, ApJ, 707, 727 Abdo, A. A., Ackermann, M., Ajello, M., et al. 2009c, ApJ, 707, L142 Ackermann, M., Ajello, M., Atwood, W. B., et al. 2015, ApJ, 810, 14 An, T., Wu, F., Yang, J., et al. 2012, ApJS, 198, 5

Angelakis, E., Fuhrmann, L., Nestoras, I., et al. 2012, J. Phys. Conf. Ser., 372, 012007

Angelakis, E., Fuhrmann, L., Marchili, N., et al. 2015, A\&A, 575, A55 Atwood, W. B., Abdo, A. A., Ackermann, M., et al. 2009, ApJ, 697, 1071 Berton, M., Foschini, L., Ciroi, S., et al. 2015, A\&A, 578, A28

Böck, M. 2012, Ph.D. Thesis, Friedrich-Alexander-Universität ErlangenNürnberg, Erlangen, Germany

Boller, T., Brandt, W. N., Fabian, A. C., \& Fink, H. H. 1997, MNRAS, 289, 393 Caccianiga, A., Antón, S., Ballo, L., et al. 2014, MNRAS, 441, 172 Caccianiga, A., Antón, S., Ballo, L., et al. 2015, MNRAS, 451, 1795 Chhetri, R., Ekers, R. D., Jones, P. A., \& Ricci, R. 2013, MNRAS, 434, 956 Dallacasa, D., Fanti, C., Giacintucci, S., et al. 2002a, A\&A, 389, 126 Dallacasa, D., Tinti, S., Fanti, C., et al. 2002b, A\&A, 389, 115 D'Ammando, F., Orienti, M., Finke, J., et al. 2012, MNRAS, 426, 317 D'Ammando, F., Orienti, M., Doi, A., et al. 2013a, MNRAS, 433, 952 D'Ammando, F., Orienti, M., Finke, J., et al. 2013b, MNRAS, 436, 191 D’Ammando, F., Larsson, J., Orienti, M., et al. 2014, MNRAS, 438, 3521
D’Ammando, F., Orienti, M., Larsson, J., \& Giroletti, M. 2015, MNRAS, 452 520

Deller, A. T., Tingay, S. J., Bailes, M., \& West, C. 2007, PASP, 119, 318

Deller, A. T., Brisken, W. F., Phillips, C. J., et al. 2011, PASP, 123, 275

Doi, A., Nagai, H., Asada, K., et al. 2006, PASJ, 58, 829

Doi, A., Nagira, H., Kawakatu, N., et al. 2012, ApJ, 760, 41

Drinkwater, M. J., Webster, R. L., Francis, P. J., et al. 1997, MNRAS, 284, 85

Fanti, C., Pozzi, F., Dallacasa, D., et al. 2001, A\&A, 369, 380

Fanti, C., Fanti, R., Zanichelli, A., Dallacasa, D., \& Stanghellini, C. 2011, A\&A, 528, A110

Fomalont, E., van Kempen, T., Kneissl, R., et al. 2014, The Messenger, 155, 19

Foschini, L. 2011, in Narrow-Line Seyfert 1 Galaxies and their Place in the Universe, PoS(NLS1)024

Foschini, L., Berton, M., Caccianiga, A., et al. 2015, A\&A, 575, A13

Freedman, W. L., Madore, B. F., Gibson, B. K., et al. 2001, ApJ, 553, 47

Gallo, L. C., Edwards, P. G., Ferrero, E., et al. 2006, MNRAS, 370, 245

Ghisellini, G., Maraschi, L., \& Tavecchio, F. 2009, MNRAS, 396, L105

Greisen, E. W. 2003, in Information Handling in Astronomy - Historical Vistas, ASSL, 285, 109

Griffith, M. R., \& Wright, A. E. 1994, VizieR Online Data Catalog: VIII/027

Großberger, C. 2014, Ph.D. Thesis, Friedrich-Alexander-Universität ErlangenNürnberg, Erlangen, Germany

Gu, M., Chen, Y., Komossa, S., et al. 2015, ApJS, 221, 3

Healey, S. E., Romani, R. W., Taylor, G. B., et al. 2007, ApJS, 171, 61

Houck, J. C., \& Denicola, L. A. 2000, in Astronomical Data Analysis Software and Systems IX, eds. N. Manset, C. Veillet, \& D. Crabtree, ASP Conf. Ser., 216,591

Hovatta, T., Nieppola, E., Tornikoski, M., et al. 2008, A\&A, 485, 51

Hovatta, T., Aller, M. F., Aller, H. D., et al. 2014, AJ, 147, 143

Kadler, M., Ojha, R., \& TANAMI Collaboration 2015, Astron. Nachr., 336, 499

Kellermann, K. I., \& Pauliny-Toth, I. I. K. 1969, ApJ, 155, L71

Kharb, P., Lister, M. L., \& Cooper, N. J. 2010, ApJ, 710, 764

Komossa, S., Voges, W., Xu, D., et al. 2006, AJ, 132, 531

Komossa, S., Xu, D., Fuhrmann, L., et al. 2015, A\&A, 574, A121

Kovalev, Y. Y., Kellermann, K. I., Lister, M. L., et al. 2005, AJ, 130, 2473

Kreikenbohm, A., Schulz, R., Kadler, M., et al. 2016, A\&A, 585, A91

Kunert-Bajraszewska, M., Gawroński, M. P., Labiano, A., \& Siemiginowska, A. 2010, MNRAS, 408, 2261

Lister, M. L., \& Homan, D. C. 2005, AJ, 130, 1389

Lister, M. L., Aller, H. D., Aller, M. F., et al. 2009, AJ, 137, 3718

Lister, M. L., Aller, M. F., Aller, H. D., et al. 2013, AJ, 146, 120

Marscher, A. P. 2009, ArXiv e-prints [arXiv:0909.2576]

Mauch, T., Murphy, T., Buttery, H. J., et al. 2013, VizieR Online Data Catalog: VIII/081

Müller, C., Kadler, M., Ojha, R., et al. 2014, A\&A, 562, A4

Murphy, T., Sadler, E. M., Ekers, R. D., et al. 2010, MNRAS, 402, 2403

O'Dea, C. P. 1998, PASP, 110, 493

O’Dea, C. P., \& Baum, S. A. 1997, AJ, 113, 148

Ojha, R., Kadler, M., Böck, M., et al. 2010, A\&A, 519, A45

Orienti, M., D'Ammando, F., Larsson, J., et al. 2015, MNRAS, 453, 4037

Oshlack, A. Y. K. N., Webster, R. L., \& Whiting, M. T. 2001, ApJ, 558, 578

Osterbrock, D. E., \& Pogge, R. W. 1985, ApJ, 297, 166

Pushkarev, A. B., \& Kovalev, Y. Y. 2012, A\&A, 544, A34

Randall, K. E., Hopkins, A. M., Norris, R. P., \& Edwards, P. G. 2011, MNRAS, 416, 1135

Readhead, A. C. S. 1994, ApJ, 426, 51

Richards, J. L., \& Lister, M. L. 2015, ApJ, 800, L8

Richards, J. L., Max-Moerbeck, W., Pavlidou, V., et al. 2011, ApJS, 194, 29

Shepherd, M. C., Pearson, T. J., \& Taylor, G. B. 1994, BAAS, 26, 987

Stanghellini, C. 2003, PASA, 20, 118

Stevens, J., Edwards, P. G., Ojha, R., et al. 2012, Fermi \& Jansky Proceedings eConf C1111101 [arXiv: 1205.2403]

Urry, C. M., \& Padovani, P. 1995, PASP, 107, 803

Wajima, K., Fujisawa, K., Hayashida, M., et al. 2014, ApJ, 781, 75

Wayth, R. B., Lenc, E., Bell, M. E., et al. 2015, PASA, 32, 25

Wilkinson, P. N., Polatidis, A. G., Readhead, A. C. S., Xu, W., \& Pearson, T. J. 1994, ApJ, 432, L87

Wright, A., \& Otrupcek, R. 1990, Parkes Catalog, Australia Telescope National Facility

Wright, A. E., Griffith, M. R., Burke, B. F., \& Ekers, R. D. 1994, ApJS, 91, 111

Yao, S., Yuan, W., Zhou, H., et al. 2015, MNRAS, 454, L16

Yuan, W., Zhou, H. Y., Komossa, S., et al. 2008, ApJ, 685, 801

Zhou, H., Wang, T., Yuan, W., et al. 2006, ApJS, 166, 128

Zhou, H., Wang, T., Yuan, W., et al. 2007, ApJ, 658, L13 
Appendix A: Data from ATCA observations

Table A.1. Details from ATCA observations.

\begin{tabular}{rcc}
\hline \hline$v$ & Date & $S_{v}$ \\
{$[\mathrm{GHz}]$} & {$[$ YYYY-MM-DD] } & {$[\mathrm{Jy}]$} \\
\hline 5.5 & $2010-02-13$ & $0.52 \pm 0.02$ \\
9.0 & $2010-02-13$ & $0.38 \pm 0.02$ \\
43.0 & $2010-05-09$ & $0.16 \pm 0.02$ \\
45.0 & $2010-05-09$ & $0.15 \pm 0.02$ \\
1.7 & $2010-07-03$ & $0.77 \pm 0.03$ \\
35.0 & $2010-07-26$ & $0.18 \pm 0.02$ \\
33.0 & $2010-07-26$ & $0.19 \pm 0.02$ \\
43.0 & $2010-09-14$ & $0.10 \pm 0.01$ \\
45.0 & $2010-09-14$ & $0.10 \pm 0.01$ \\
2.1 & $2010-12-17$ & $0.67 \pm 0.03$ \\
33.0 & $2011-04-12$ & $0.16 \pm 0.02$ \\
35.0 & $2011-04-12$ & $0.16 \pm 0.02$ \\
5.5 & $2011-05-17$ & $0.41 \pm 0.02$ \\
9.0 & $2011-05-17$ & $0.29 \pm 0.01$ \\
17.0 & $2011-05-17$ & $0.18 \pm 0.01$ \\
19.0 & $2011-05-17$ & $0.17 \pm 0.01$ \\
5.5 & $2011-10-14$ & $0.41 \pm 0.02$ \\
9.0 & $2011-10-14$ & $0.29 \pm 0.01$ \\
17.0 & $2011-10-14$ & $0.18 \pm 0.01$ \\
19.0 & $2011-10-14$ & $0.16 \pm 0.01$ \\
5.5 & $2011-11-08$ & $0.40 \pm 0.02$ \\
9.0 & $2011-11-08$ & $0.26 \pm 0.01$ \\
17.0 & $2011-11-08$ & $0.18 \pm 0.01$ \\
19.0 & $2011-11-08$ & $0.16 \pm 0.01$ \\
38.0 & $2011-11-08$ & $0.13 \pm 0.01$ \\
40.0 & $2011-11-08$ & $0.13 \pm 0.01$ \\
5.5 & $2011-11-27$ & $0.37 \pm 0.01$ \\
9.0 & $2011-11-27$ & $0.28 \pm 0.01$ \\
17.0 & $2011-11-27$ & $0.20 \pm 0.01$ \\
19.0 & $2011-11-27$ & $0.18 \pm 0.01$ \\
5.5 & $2012-01-15$ & $0.38 \pm 0.02$ \\
9.0 & $2012-01-15$ & $0.25 \pm 0.01$ \\
5.5 & $2012-03-17$ & $0.41 \pm 0.02$ \\
9.0 & $2012-03-17$ & $0.27 \pm 0.01$ \\
5.5 & $2012-04-22$ & $0.33 \pm 0.01$ \\
9.0 & $2012-04-22$ & $0.19 \pm 0.01$ \\
17.0 & $2012-05-28$ & $0.15 \pm 0.01$ \\
19.0 & $2014-03-26$ & $0.28 \pm 0.01$ \\
19.0 & $2012-05-28$ & $0.14 \pm 0.01$ \\
38.0 & $2012-05-28$ & $0.09 \pm 0.01$ \\
40.0 & $2012-05-28$ & $0.09 \pm 0.01$ \\
5.5 & $2012-06-13$ & $0.41 \pm 0.02$ \\
9.0 & $2012-06-13$ & $0.29 \pm 0.01$ \\
17.0 & $2012-06-13$ & $0.16 \pm 0.01$ \\
19.0 & $2012-06-13$ & $0.14 \pm 0.01$ \\
38.0 & $2012-06-13$ & $0.08 \pm 0.01$ \\
40.0 & $2012-06-13$ & $0.08 \pm 0.01$ \\
17.0 & $2012-09-07$ & $0.19 \pm 0.01$ \\
19.0 & $2012-09-07$ & $0.17 \pm 0.01$ \\
5.5 & $2012-11-02$ & $0.45 \pm 0.02$ \\
9.0 & $2012-11-02$ & $0.33 \pm 0.01$ \\
5.5 & $2013-11-10$ & $0.44 \pm 0.02$ \\
9.0 & $2013-11-10$ & $0.31 \pm 0.01$ \\
5.5 & $2014-03-25$ & $0.49 \pm 0.02$ \\
5.5 & $2014-03-25$ & $0.30 \pm 0.01$ \\
\hline & $2014-03-26$ & $0.56 \pm 0.02$ \\
\hline .0 & & \\
\hline
\end{tabular}

